$X-614-65-202$

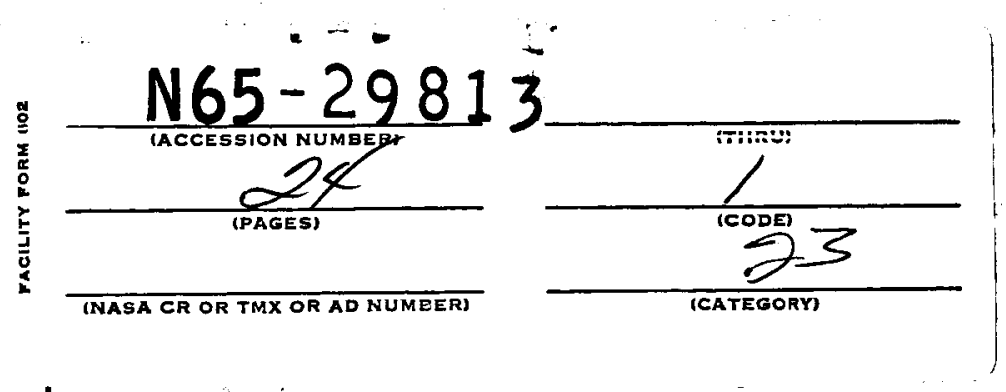

\title{
THE REFLECTION OF SOFT X-RAYS, BY POLISHED SURFACES OF GLASS AND STEEL
}

\section{BY \\ JAMES H. UNDERWOOD}

GPO PRICE

$\$$

CFSTI PRICE(S) \$

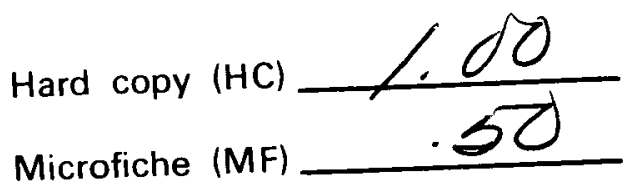

ff 653 July 65

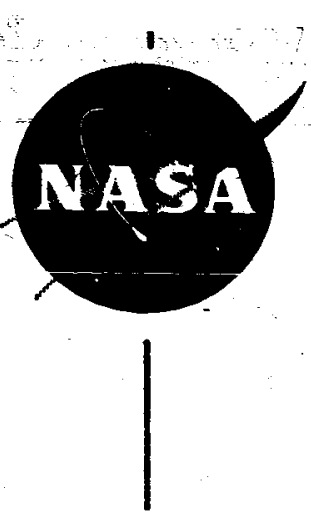




\title{
THE REFLECTION OF SOFT X-RAYS BY POLISHED \\ SURFACES OF GLASS AND STEEL
}

By

\author{
J. H. Underwood \\ University of Leicester, England*
}

\section{ABSTRACT}

$298 / 3$

Using a vacuum reflectometer which employs a mica crystal monochromator and a proportional counter detector, measurements of the efficiency of reflection of soft X-rays by optical flats of "Pyrex" glass and stainless steel were made at a number of wavelengths in the region 5 - 16 \&. Values of the refractive index and mass absorption coefficient of the media were obtained from the curves of reflection efficiency versus glancing angle, and compared with predicted values. A disagreement between theory and experiment was found, which could be attributed to microscopic surface roughness, and a reduced surface electron density.

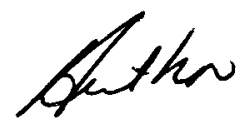

*Now at Solar Physics Branch, Goddard Space Flight Center, National Aeronautics and Space Administration, Greenbe1t, Maryland, USA, under an NAS/NSF postdoctoral research associateship. 


\section{INTRODUCTION}

Recent developments in the fields of $\mathrm{X}$-ray astronomy and microscopy have lead to a revival of interest in the technique of glancing incidence "total" reflection as a means of producing X-ray images. In both applications, the soft ( $5-50 \AA) \mathrm{X}$-ray region is of great importance, but at the present time there is a lack of accurate experimental data on the efficiency of reflection of various materials in this wavelength region. A certain amount is available from the work of pre-war experimenters (see Compton and Allison (1) for a complete list of references) but most of these results were obtained using photographic film for the measurement of intensity, a method which can lead to serious errors owing to the non-linearity of X-ray film in this region, and the difficulty of intensity calibration. The only investigations carried out in the soft $X-r$ ay region using modern photoncounting techniques have been those of Hendrick (5) at $8.3 \AA$ and Wuerker (13) at $44.6 \mathrm{R}$. The work described in this paper was undertaken in an attempt to fill in some of the gaps in the reflection data, to investigate the suitability of various surfaces as $X$-ray imaging mirrors, and to test the theories of reflection and dispersion of X-rays in the soft X-ray region.

\section{THEORY}

As the refractive index, $n=1-\delta$, of all materials is slightly less than unity throughout most of the X-ray region, X-rays incident on a surface from vacuum at angles of incidence approaching $90^{\circ}$ will be totally externally reflected. There is, associated with this reflection, a critical angle of glancing incidence $\theta_{c}\left(\theta=90^{\circ}\right.$ - optical angle of incidence) given by the equation 


$$
\operatorname{Cos} \theta_{c}=n=1-\delta
$$

which, for the small values of $\hat{\theta}$ which are involved, may be written

$$
\theta_{c}=\sqrt{2 \delta}
$$

The reflection is not, in fact, total, as some absorption by the medium always takes place, thereby reducing the intensity of the reflected beam. Nor is there a sharp cut-off at the critical angle, except when the absorption by the medium is very small. For high absorption (as is usually the case in the soft $\mathrm{X}$-ray region) the reflected intensity decreases more or less gradually from $\theta=0$ output past the critical angle given by equation (2). For a medium whose linear absorption co-efficient is $\mu_{\ell}$, the variation with $\theta$ of $\frac{I}{I_{0}}$, the ratio of the reflected to the incident intensity, is given by the modified Fresnel equation:

$$
\frac{I}{I_{0}}=\frac{\left[2 x-\sqrt{\sqrt{\left(x^{2}-1\right)^{2}+y^{2}}+\left(x^{2}-1\right)}\right]^{2}+\sqrt{\left(x^{2}-1\right)+y^{2}}-\left(x^{2}-1\right)}{\left[2 x+\sqrt{\sqrt{\left(x^{2}-1\right)^{2}+y^{2}}+\left(x^{2}-1\right)}\right]^{2}+\sqrt{\left(x^{2}-1\right)+y^{2}}-\left(x^{2}-1\right)} \ldots \text { (3) }
$$

where $\mathrm{x}=\theta / \theta_{c}, \quad Y=\mu_{e} \lambda / 4 \pi \delta$

If a curve of the type given by equation (3) can be fitted to an experimentally determined curve of $\frac{I_{1}}{I_{0}}$ versus $\theta$, values of the parameters $Y$ and $\theta_{c}$, and thus of $\mu_{e}$ and $\delta$ can be obtained. The values of $\mu_{e}$ may be compared with directly determined values of the absorption coefficient, and the values of $\delta$ with those obtained from the dispersion theories of Drude-Lorentz, Kallmann gnd Mark (8), Hön1 (7) or Perratt and Hempstead (11). In this way the validity of equation (3) for the soft $\mathrm{X}$-ray region, and of the dispersion theories, may be tested. 


\section{APPARATUS}

A special vacuum reflectometer was constructed for this work, and a schematic diagram of the instrument is shown in Fig. 1. Characteristic X-radiation, produced by a conventional X-ray source with a demountable target, was isolated by the mica monochromator and limited to a narrow beam by the two slits. Each slit considted of two pieces of 5/16" dia. silver steel rod, etched with acid to prevent their giving troublesome reflections, and separated by spacers. The beam was allowed to fall on the reflecting specimen, which was mounted on a rotating table carrying a vernier calibrated in minutes of arc, by means of which the angular position of the specimen could be read off. The scale was calibrated to read glancing angle by a photographic method. By means of a motor, the specimen could be withdrawn from the beam and accurately repositioned. In this way the intensity of the direct beam could be measured. Intensities were measured with a flow proportional counter having a thin (less than one micron) "Melinex" window. The counter pulses were amplified with a high gain amplifier and counted with a conyentional scaler. The resolution of the counter was sufficient to allow discrimination, by single channel pulse height analysis, against very soft radiation totally reflected from the mica crystal, and hard radiation produced by second and higher orders of Bragg reflection. This meant that the source could be run at a voltage several times that required to excite the particular line being used, so that a high intensity could be obtained.

The reflectometer was enclosed in a cylindrical vacuum tank of machined aluminium, and the reflection measurements were made at a pressure 


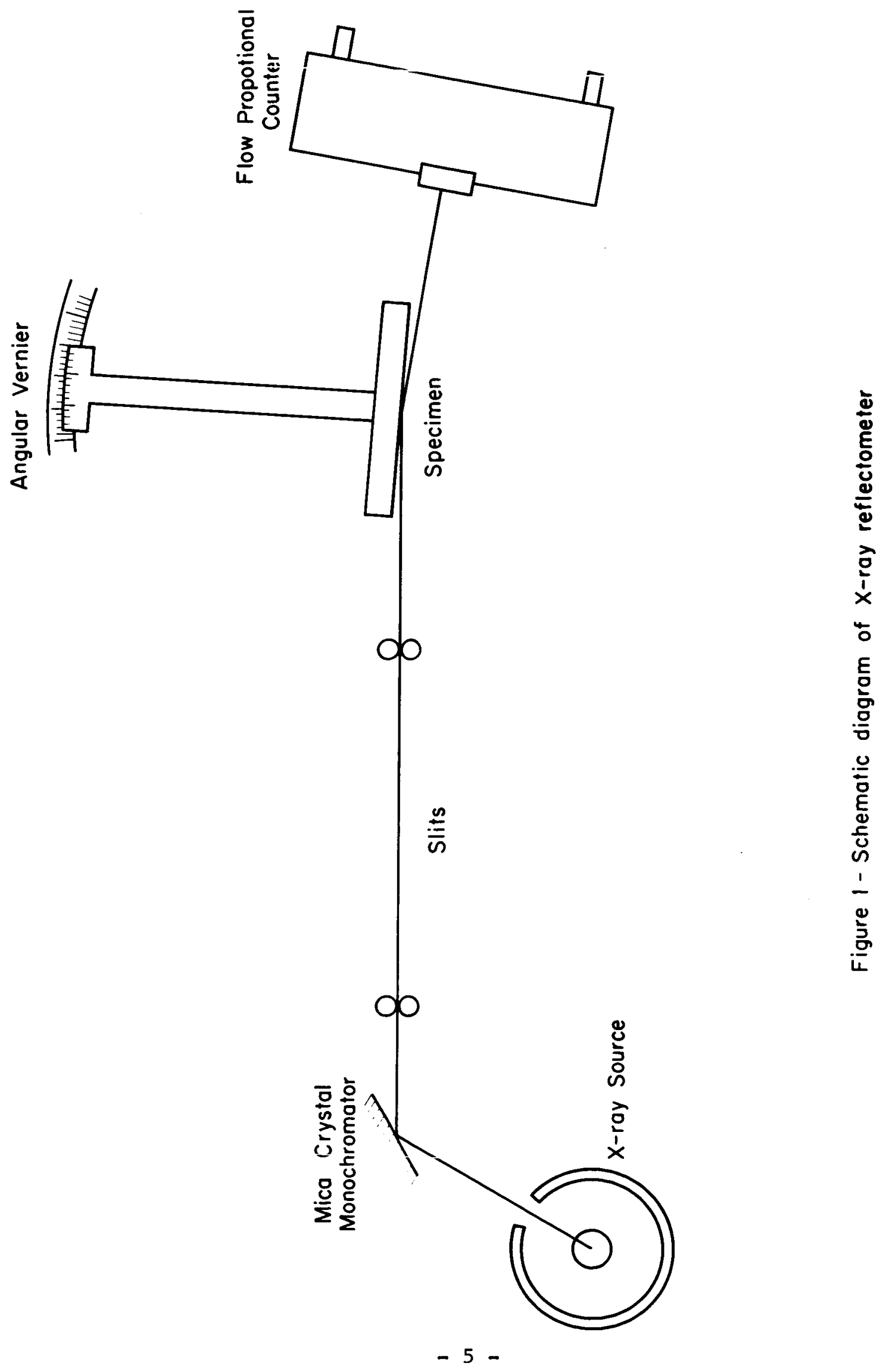


of around $10^{-5}$ torr.

\section{EXPERTMENTAL PROCEDURE}

Characteristic lines of elements were obtained by using a target of a material containing the element in question or by spreading a paste of a compound (usually the oxide) of the element on the surface. Originally it was hoped that measurements could be made up to the wavelength limit set by the mica crystal (about $20 \AA$ ), but absorption by the counter window limited the range of measurements to the wavelength of Co $L$ radiation $(16.00 \AA)$

Curves of $\frac{I}{I_{0}}$ versus $\theta$ were taken at a number of wavelengths for each specimen, by taking repeated measurements of the intensity in the direct and reflected beams at different values of the glancing angle. The measurements were taken by a method due to Nordfors (9), in order to compensate for small drifts in emission. The total number of counts accumulated in a measurement of the direct beam intensity was typically of the order of 20,000 . Theoretical curves of the type given by equation (3) were fitted to each experimental curve for $\frac{I}{I_{0}}$, the fitting being done by a least squares method asing an E1liot 803 computer.

\section{RESULTS}

Extensive measurements in the wavelength region $5-16 \&$ were made on optical flats of "Pyrex" glass and stainless steel. The flats, having dimensions $2 " x 1 " \times 3 / 8 "$, were produced by conventional optical polishing methods, and were claimed by the makers to be flat to within $1 / 10$ wavelength of visible (green) light. The results are plotted in Figures $2-6 . \frac{I}{\bar{I}_{0}}$ 
values are tabulated in lables 1 and $\bar{z}$, and vaiues of $y$ ănu $\hat{\theta}_{c}$, obtained from the fitting of Fresnel curves, in Tables 3 and 4.

\section{EXPERIMENTAL ERROR}

Most of the uncertainty in the values of $\frac{I}{I_{0}}$ is due to the statistical error in counting. This was estimated to be $\pm 3 \%$, except for those values of $\frac{I}{I_{0}}$ below about 0.07 , for which the intensity in the reflected beam was lower, and thus the statistical error higher. The uncertainty in these values may be as high as $\pm 10 \%$.

The values of $\frac{I}{I_{0}}$ for 14.6 and $16.0 \&\left(N_{i} h_{\alpha}\right.$ and $\left.C_{0} L_{\alpha}\right)$ radiations also have a larger statistical error, as window absorption reduced the count rate in both the direct and the reflected beams. The error in the values of $\frac{I}{\bar{I}_{0}}$ for these radiations is estimated to be approximately twice the corresponding error for the harder X-rays.

The uncertainty in the measurement of glancing angle is \pm .2 minutes of arc. In addition to this, the beam diverged by 3 minutes but, as pointed out by Hendrick ( $l \circ c$. cit.) this affects only the angular resolution in determining the $\frac{I}{I_{0}}$ curves, not the angular accuracy. At the long wavelengths under investigation, the effect on the curve shapes is negligible.

\section{DISCUSSION}

From Figures $2-6$, we see that equation (3) describes the reflection of $X$-rays remarkably well, in view of the fact that, in its original form, it was derived over 150 years ago, on the basis of the elastic-solid theory of light. However, in many of the curves, the experimental points lie below 
TABLE 1

Reflectivity $\frac{I}{\bar{I}_{0}}$ of Pyrex glass at various wavelengths

\begin{tabular}{|c|c|c|c|c|c|c|c|c|c|c|c|}
\hline$\theta_{(\min )}$ & $\begin{array}{r}\mathrm{P} \mathrm{K} g \\
6.16 \AA\end{array}$ & $\begin{array}{l}\text { SrLP } \\
6.62 \AA\end{array}$ & $\begin{array}{l}\text { W MB } \\
6.76 \AA\end{array}$ & $\begin{array}{l}\text { SrIx } \\
6.86 \AA\end{array}$ & $\begin{array}{l}\mathrm{A} \downarrow \mathrm{K}_{\mathrm{ab}} \\
8.34 \AA\end{array}$ & $\begin{array}{l}\mathrm{MgK} \\
9.89 \AA\end{array}$ & $\begin{array}{l}\text { GeI } \boldsymbol{\alpha} \\
11.31 \AA\end{array}$ & $\begin{array}{l}\mathrm{Za} I a \\
12.28\end{array}$ & $\begin{array}{l}\text { CuL } 1 \\
13.3 \AA\end{array}$ & $\begin{array}{l}\text { Ni Iod } \\
14.6 \AA\end{array}$ & $\begin{array}{l}\text { Gole } \\
16.0 \AA\end{array}$ \\
\hline 33 & .743 & - & - & .897 & .878 & .882 & - & .848 & - & - & - \\
\hline 38 & .740 & .698 & .826 & .870 & .883 & .842 & - & .797 & .780 & .727 & \\
\hline 43 & .592 & .670 & .764 & .835 & .850 & .848 & .827 & .819 & .763 & .743 & .689 \\
\hline 48 & .431 & .574 & .664 & .779 & .814 & .824 & - & - & - & - & - \\
\hline 50 & - & - & .464 & - & - & - & - & - & - & - & - \\
\hline 53 & .136 & .333 & .310 & .530 & .777 & .805 & .774 & .776 & .728 & .708 & .605 \\
\hline 55 & - & - & - & .252 & - & - & - & - & - & - & - \\
\hline 58 & .060 & .145 & .083 & .149 & .699 & .742 & - & - & - & - & - \\
\hline 63 & .031 & .079 & .040 & .078 & .619 & .731 & .721 & .732 & .682 & .717 & .538 \\
\hline 65 & - & - & - & - & .460 & - & - & - & - & - & - \\
\hline 68 & - & .042 & .025 & .042 & .271 & .652 & - & - & - & - & - \\
\hline 73 & - & .029 & - & .030 & .169 & .610 & .680 & .666 & .618 & .634 & .458 \\
\hline 78 & - & - & - & - & .076 & .485 & .591 & - & - & - & - \\
\hline 83 & - & - & - & - & .043 & .220 & .519 & .566 & .546 & .541 & .439 \\
\hline 88 & - & - & - & - & - & - & .408 & - & - & - & - \\
\hline 93 & - & - & - & - & .017 & .068 & .306 & .424 & .478 & .500 & .439 \\
\hline 98 & - & - & - & - & - & - & - & .335 & .397 & - & - \\
\hline 103 & - & - & - & - & - & .033 & .104 & .235 & .312 & .420 & .309 \\
\hline 108 & - & - & - & - & - & - & - & .143 & .212 & - & - \\
\hline 113 & - & - & - & - & - & .014 & .039 & .076 & .157 & .258 & .212 \\
\hline 118 & - & - & - & - & - & - & - & - & .080 & - & - \\
\hline 123 & - & - & - & - & - & - & .021 & .034 & .069 & .140 & .140 \\
\hline 133 & - & - & - & - & - & - & - & .021 & .034 & .069 & .074 \\
\hline 143 & - & - & - & - & - & - & - & - & - & .035 & .034 \\
\hline
\end{tabular}


Reflectivity $\frac{I}{I_{0}}$ of stainless steel at various wavelengths,

\begin{tabular}{|c|c|c|c|c|c|c|c|c|}
\hline (min) & $\begin{array}{r}N T \mathrm{~T}=0 \\
5.73 \AA\end{array}$ & $\begin{array}{l}\text { Sik } \\
\text { p.158 }\end{array}$ & $\begin{array}{l}A I \bar{K} \\
8.348\end{array}$ & $\begin{array}{l}\mathrm{MgK} \propto \\
9.89 \AA\end{array}$ & $\begin{array}{l}\text { ZnL } \\
12.28 \AA\end{array}$ & $\begin{array}{l}\text { CuL } \alpha \\
13.3 \AA\end{array}$ & $\begin{array}{l}\text { NeI } \alpha \\
14.6 \AA\end{array}$ & $\begin{array}{l}\mathrm{C}_{0} \mathrm{~L} \alpha \bar{\alpha} \\
16.0 \AA\end{array}$ \\
\hline 32 & .897 & 902 & .912 & .904 & .868 & .862 & .836 & .782 \\
\hline 42 & .840 & 853 & .871 & .860 & .826 & .818 & .788 & .767 \\
\hline 52 & .752 & 798 & .821 & .836 & .785 & .778 & .746 & - \\
\hline 57 & - & - & - & - & - & - & - & .704 \\
\hline 62 & .632 & .733 & .778 & .786 & .744 & .745 & .700 & - \\
\hline 67 & .526 & - & - & - & - & - & - & - \\
\hline 72 & .435 & .624 & .724 & .732 & .700 & .667 & .655 & .644 \\
\hline 77 & .293 & .577 & - & - & - & - & - & - \\
\hline 82 & .120 & .488 & .630 & .662 & .647 & .638 & .584 & - \\
\hline 87 & .051 & .423 & - & - & - & - & - & .563 \\
\hline 92 & .016 & .332 & .511 & .587 & .563 & .528 & .529 & - \\
\hline 102 & - & .155 & .385 & .488 & .504 & .518 & .448 & .457 \\
\hline 112 & - & .036 & .234 & .375 & .415 & .427 & .394 & - \\
\hline 117 & - & - & - & - & - & - & - & .361 \\
\hline 122 & - & 012 & 128 & 300 & 363 & 371 & 331 & 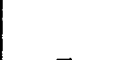 \\
\hline & & & & & . & $e^{0}$ & • & - \\
\hline 132 & - & - & .037 & .184 & .278 & .295 & .281 & .319 \\
\hline 142 & - & - & .008 & .081 & .187 & .212 & .200 & - \\
\hline 147 & - & - & - & - & - & - & - & .220 \\
\hline 152 & - & - & - & .031 & .137 & .160 & 159 & - \\
\hline 162 & - & - & - & .014 & .085 & .115 & .117 & .157 \\
\hline 172 & - & - & _ & - & 050 & 075 & 075 & - \\
\hline & & & & & & & & \\
\hline 177 & - & - & - & - & - & - & - & .090 \\
\hline 182 & - & - & - & - & .025 & .045 & .047 & - \\
\hline 192 & - & - & - & - & - & .028 & .030 & .060 \\
\hline
\end{tabular}



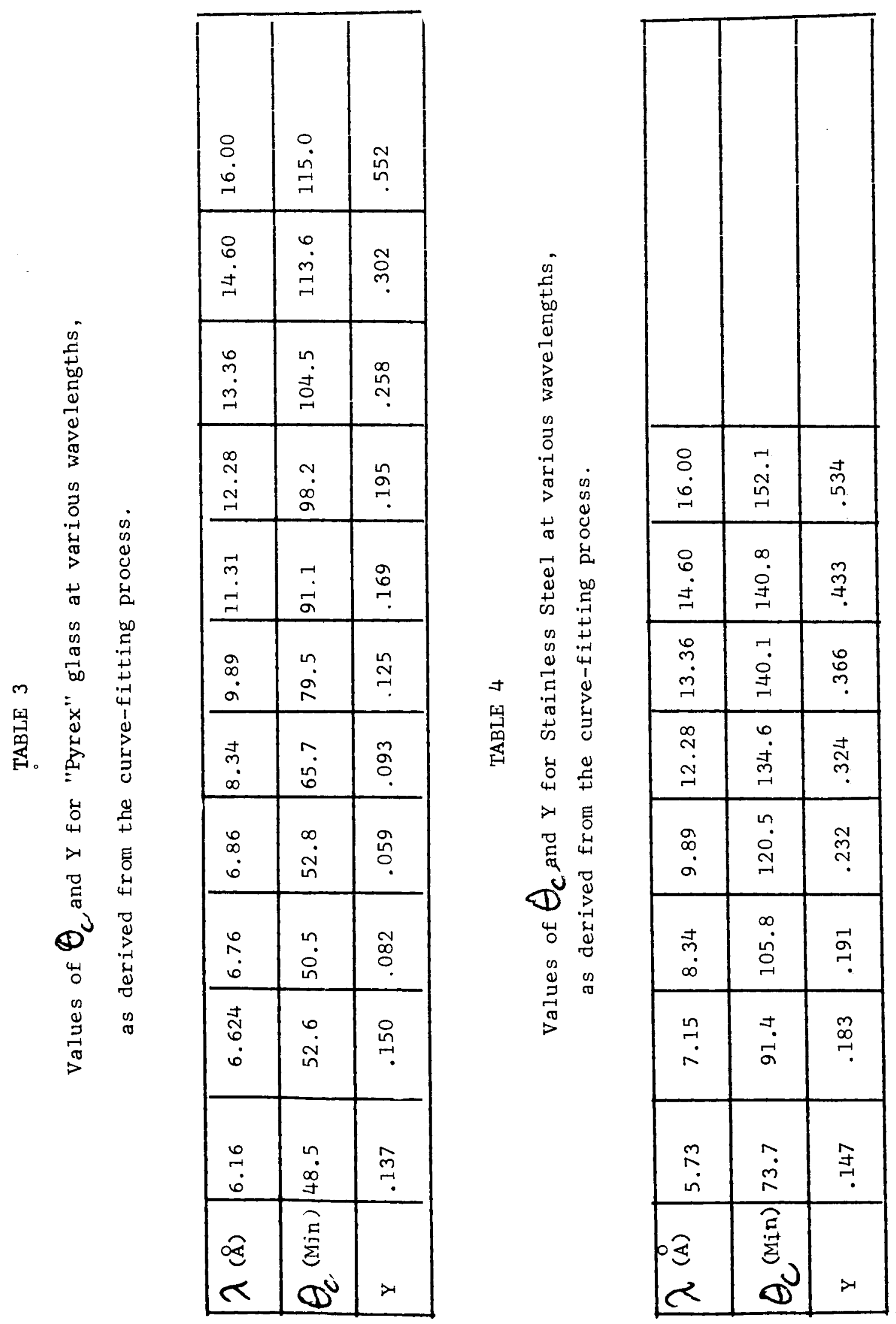


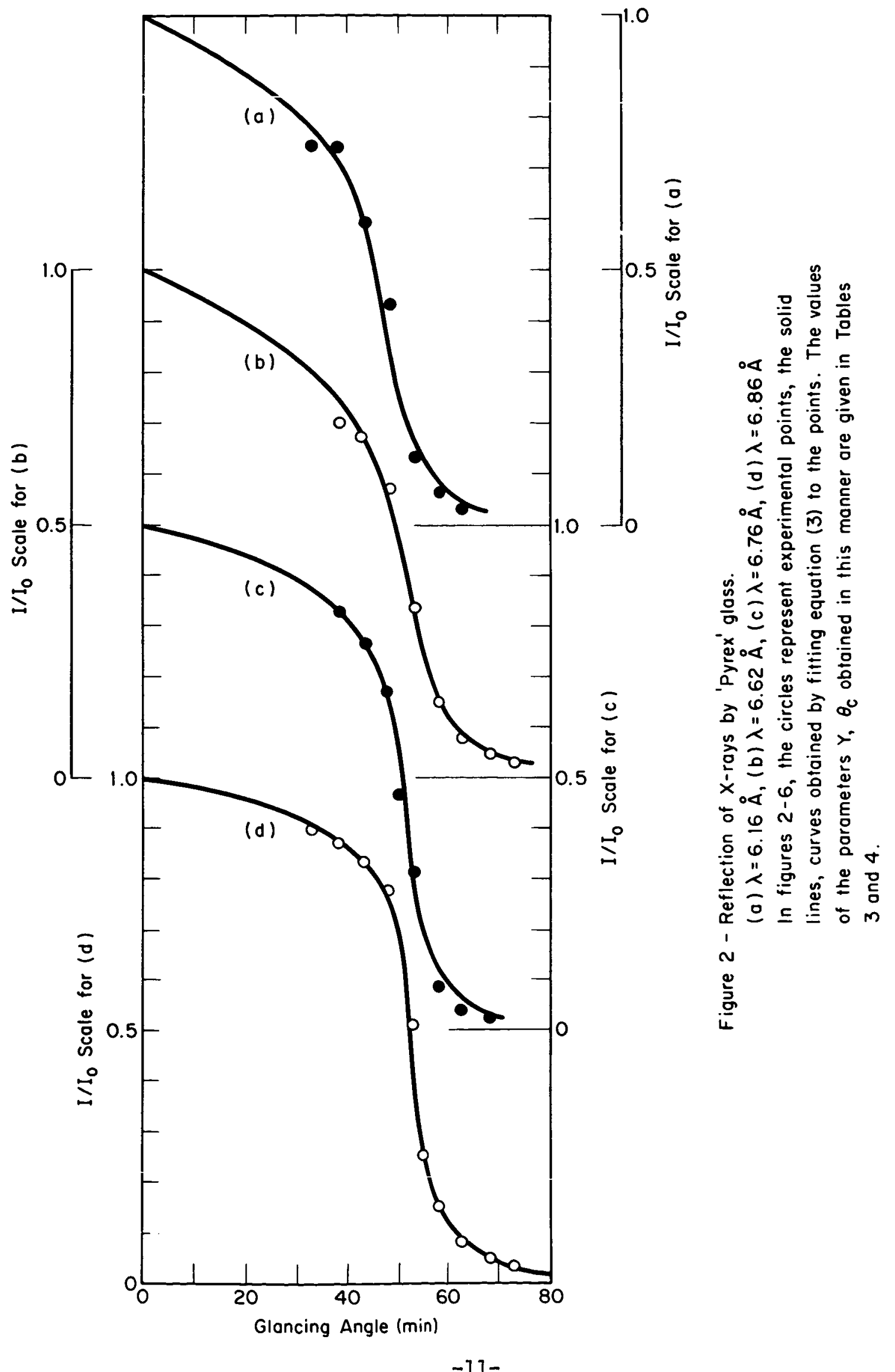




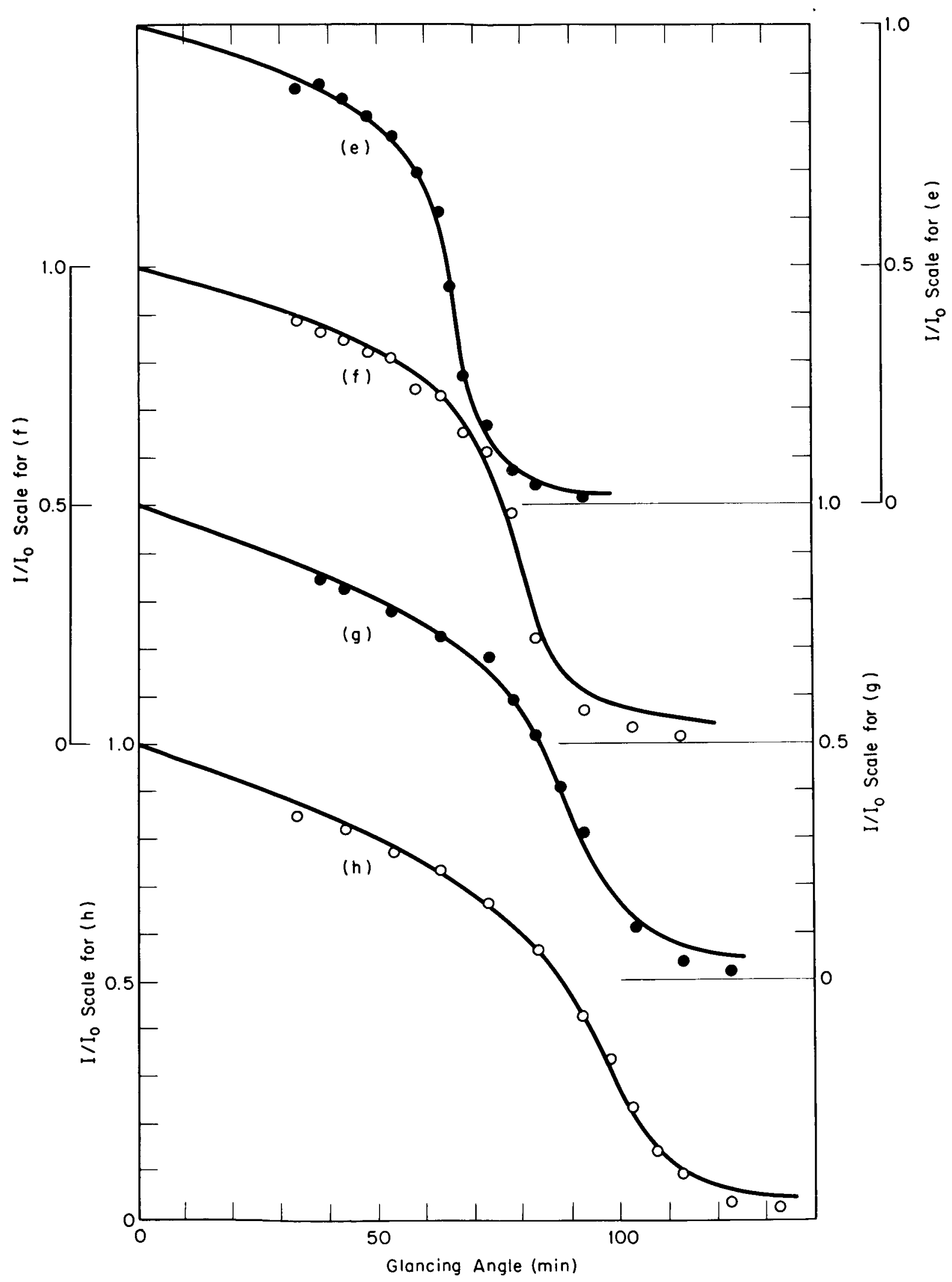

Figure 3 - Reflection of $X$-rays by 'Pyrex' glass.

(e) $\lambda=8.34 \AA,(f) \lambda=9.89 \AA,(g) \lambda=11.31 \AA,(h) \lambda=12.28 \AA$ 


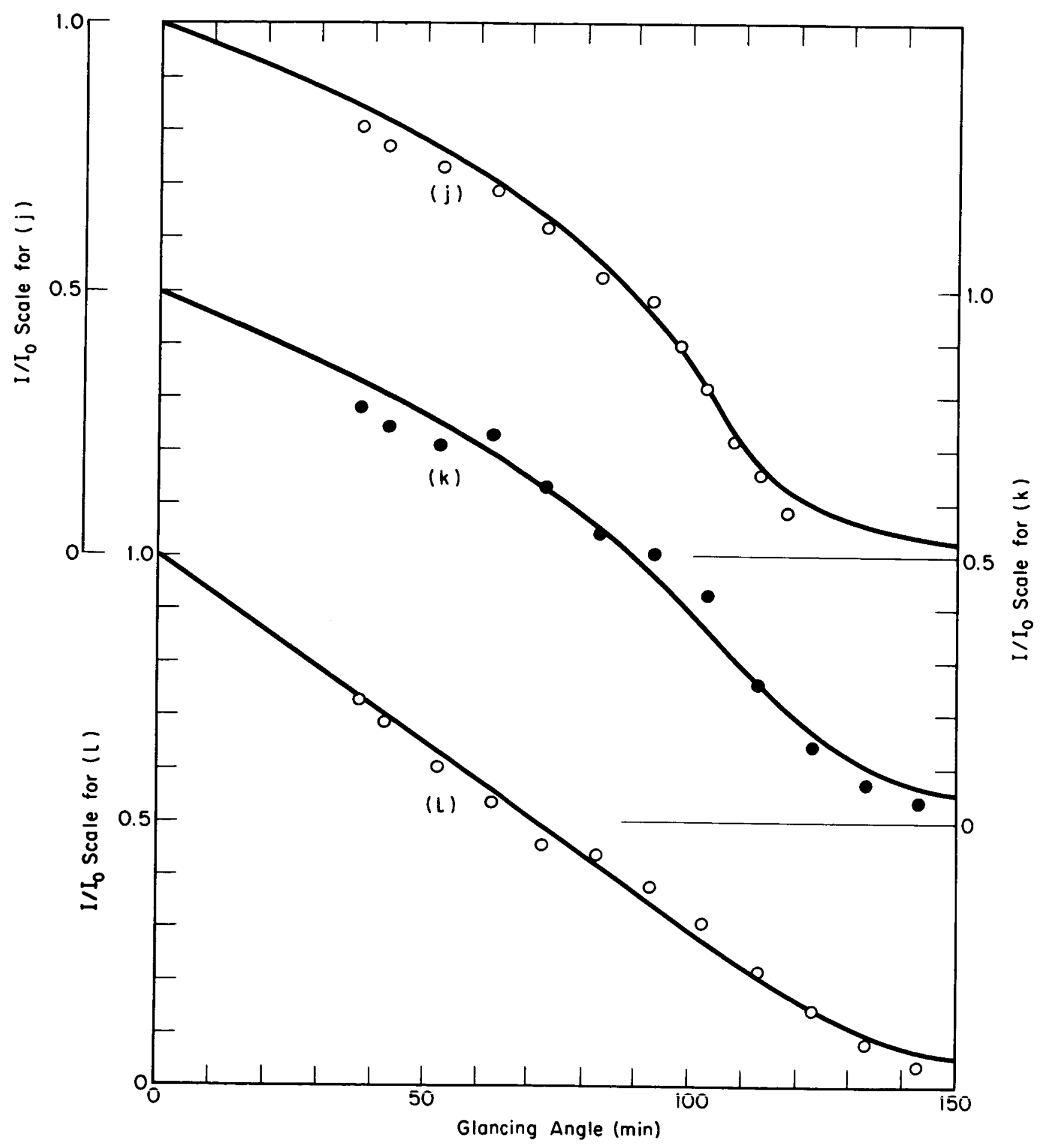

Figure 4 - Reflection of $X$-rays by 'Pyrex' glass.

(j) $\lambda=13.3 \AA$ \&, (k) $\lambda=14.6 \AA$, (l) $\lambda=16.0 \AA$ 


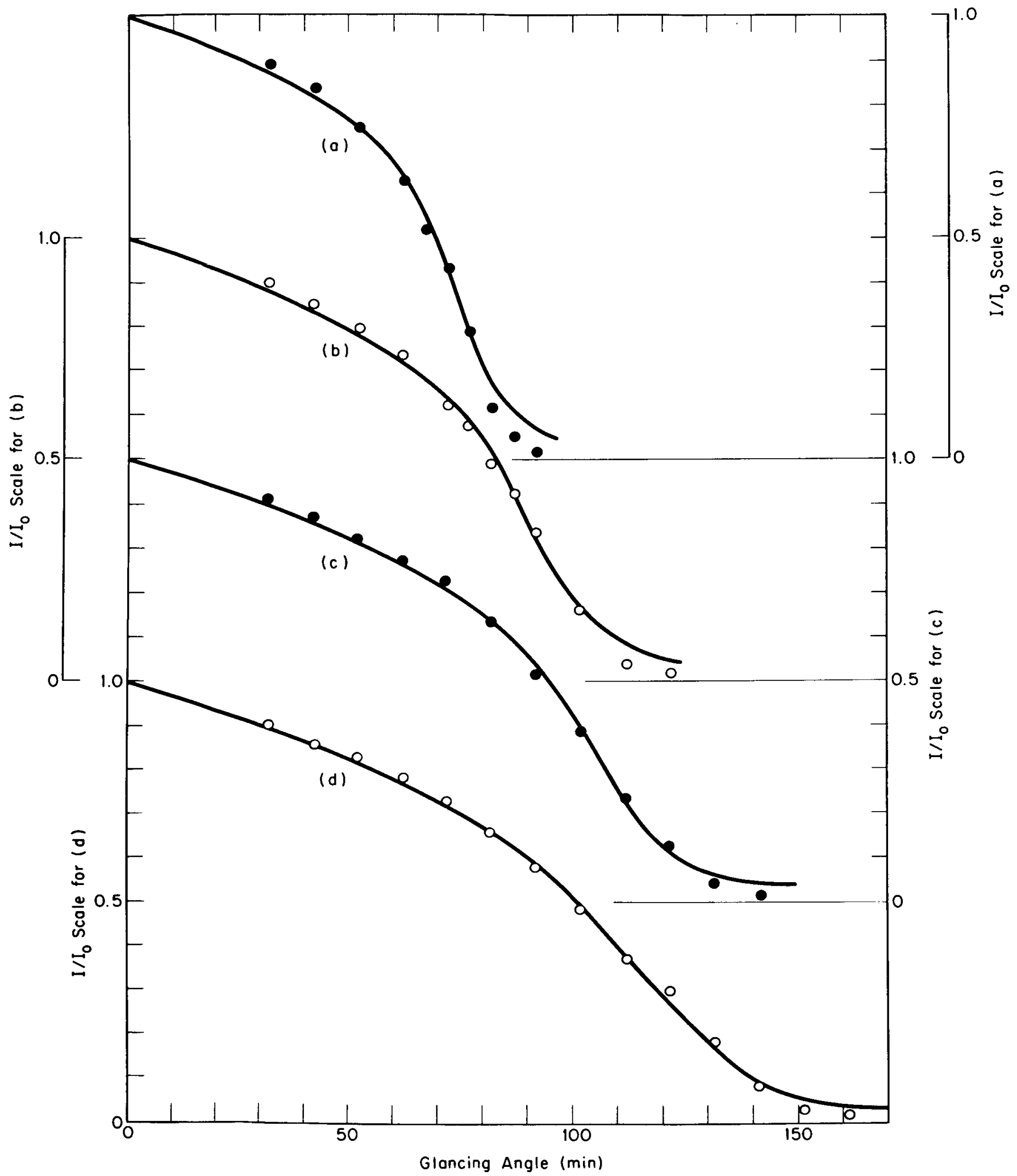

Figure 5 - Reflection of $X$-rays by Stainless Steel.

(a) $\lambda=5.75 \AA$, (b) $\lambda=7.15 \AA$, (c) $\lambda=8.34 \AA$, (d) $\lambda=9.89 \AA$ 


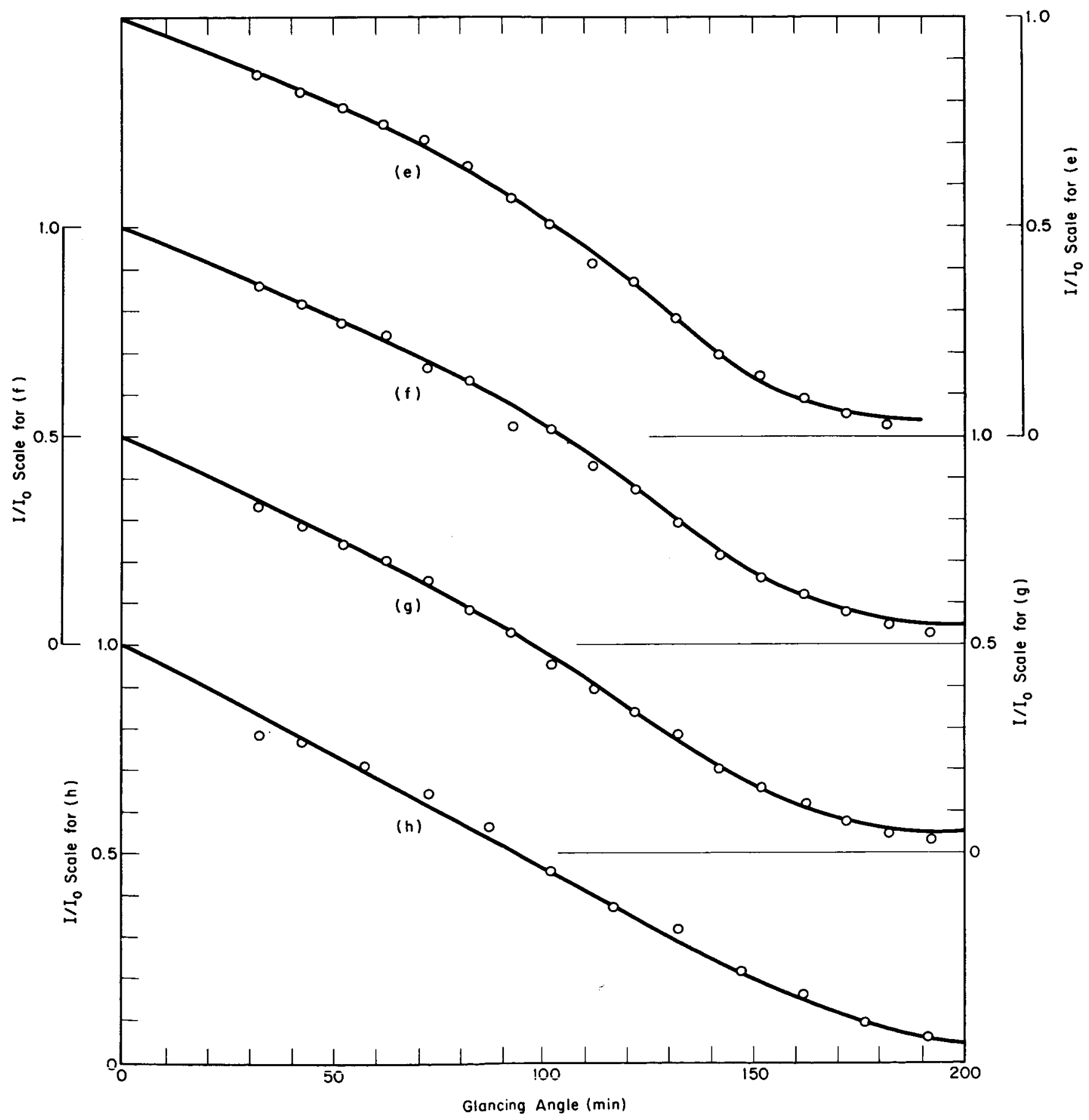

Figure 6 - Reflection of $X$-roys by Stainless Steel.

(e) $\lambda=12.28 \AA,(f) \lambda=13.3 \AA,(g) \lambda=14.6 \AA,(h) \lambda=16.0 \AA$ 
the theoretical curve at angles greater than the critical angle. A similar phenomenon was observed by Parratt (10) in experiments at shorter wavelengths on the reflection of X-rays from surfaces of oxidized copper. He attributed it to the presence of an electron density minimum at a depth of $100 \AA$ or so below the surface, which led to a "trapping" of the reflected radiation at angles of grazing incidence greater than critical. Microscopic surface roughness was discounted as a cause for the effect.

In the case of the experiments described here, surface roughness cannot be discounted as a possible cause for the discrepancy between the experimental and theoretical reflection curves. That the surface was, in fact, imperfectly smooth for X-ray reflection was shown by a series of tests using photographic film as the detector. First the direct beam, then the reflected beam were allowed to fall on a photographic plate at a distance of about $20 \mathrm{~cm}$. from the reflector. When developed, the direct beam produced a line on the film with perfectly sharp edges, but the line produced by the reflected beam had blurred edges and was flanked by a slight halation. Both the glass and stainless steel specimens gave similar results but the reflection from an evaporated copper film was much more diffuse, although in all three cases there was no degradation in the reflection of visible light. The same effect has been observed by Ehrenberg (3) by Groth (4) and, more recently, by Sauro et. al (12). The probable explanation for the diffuseness is that the surface is rough on a submicroscopic scale, so that the $X$-rays are scattered, whereas the longer wavelength, visible light, sees a smooth surface, and so is specularly reflected. It is obvious that such a rough structure would yield a reflection curve rather different from one calculated from equation (3), which is based on a model assuming a perfectly smooth surface. 
Disagreement between theory and experiment is also found when Lìe values of $\mu_{t}$ and $\delta$ ohteined from the experiments are compared with theoretical values. Figures 7 and 8 show, on a logarithmic scale, the values of $\frac{M l}{\rho}$, the mass absorption coefficient, as obtained from the values of $\mathrm{Y}$ given in Tables 3 and $4\left(\frac{\mu_{l}}{\rho}=\frac{4 \pi \theta_{c}^{2} Y}{2 \lambda \rho}\right.$, compared with those obtained by Henke et. al. (6) using a semi-empirical method.

The results for glass (Fig. 7) show fairly good agreement with the predicted values. The difference in slope of the two curves can be accounted for by experimental error and the fact that there is some uncertainty in the values of $\frac{\mu}{e}$ obtained by the semi-empirical method (Cooke, 1963 ). For steel, the agreement is not as good, and the discrepancy is probably too great to be explained by the above-mentioned factors. The results seem, however, to indicate the presence of a surface layer of reduced electron density, which would be consistent with the presence of a layer of oxide on the steel. As the difference between the experimental and the theoretical values of $\frac{\mu}{e}$ increases with wavelength (and thus with decreasing depth of penetration of $X$-rays), it would seem that the electron density is decreasing monotonically toward the extreme surface, and that there is no "reflection trap" in this case.

This conclusion would appear to be supported by the comparison of experimental and theoretical values of $\delta$ for steel (Fig. 10). The theoretical values in this case are calculated using the dispersion theory of Kallmann and Mark. It can be seen that the experimental values of fall progressively further and further below the predicted ones as longer wavelengths are approached, indicating a decreasing surface electron density. 


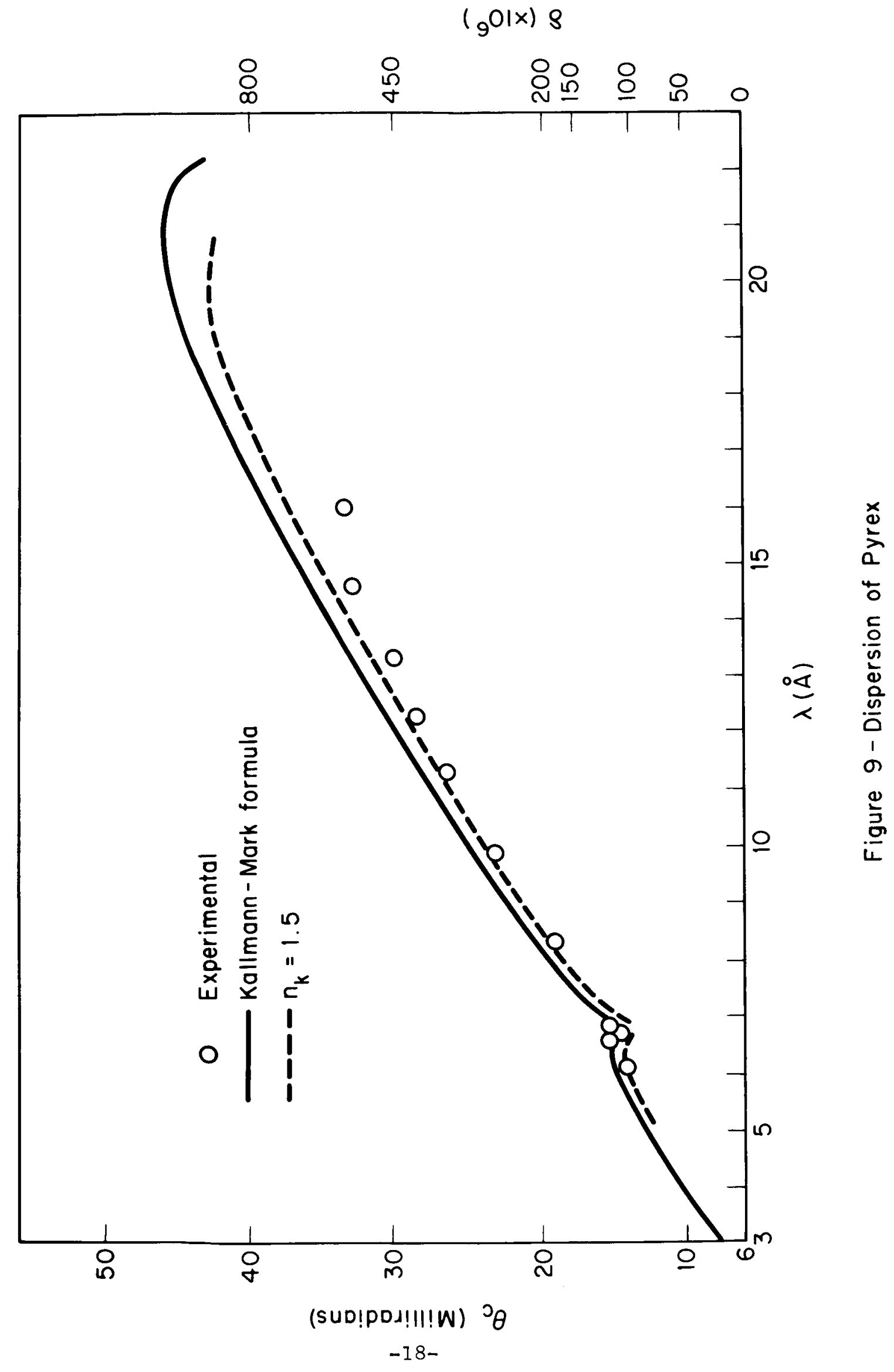




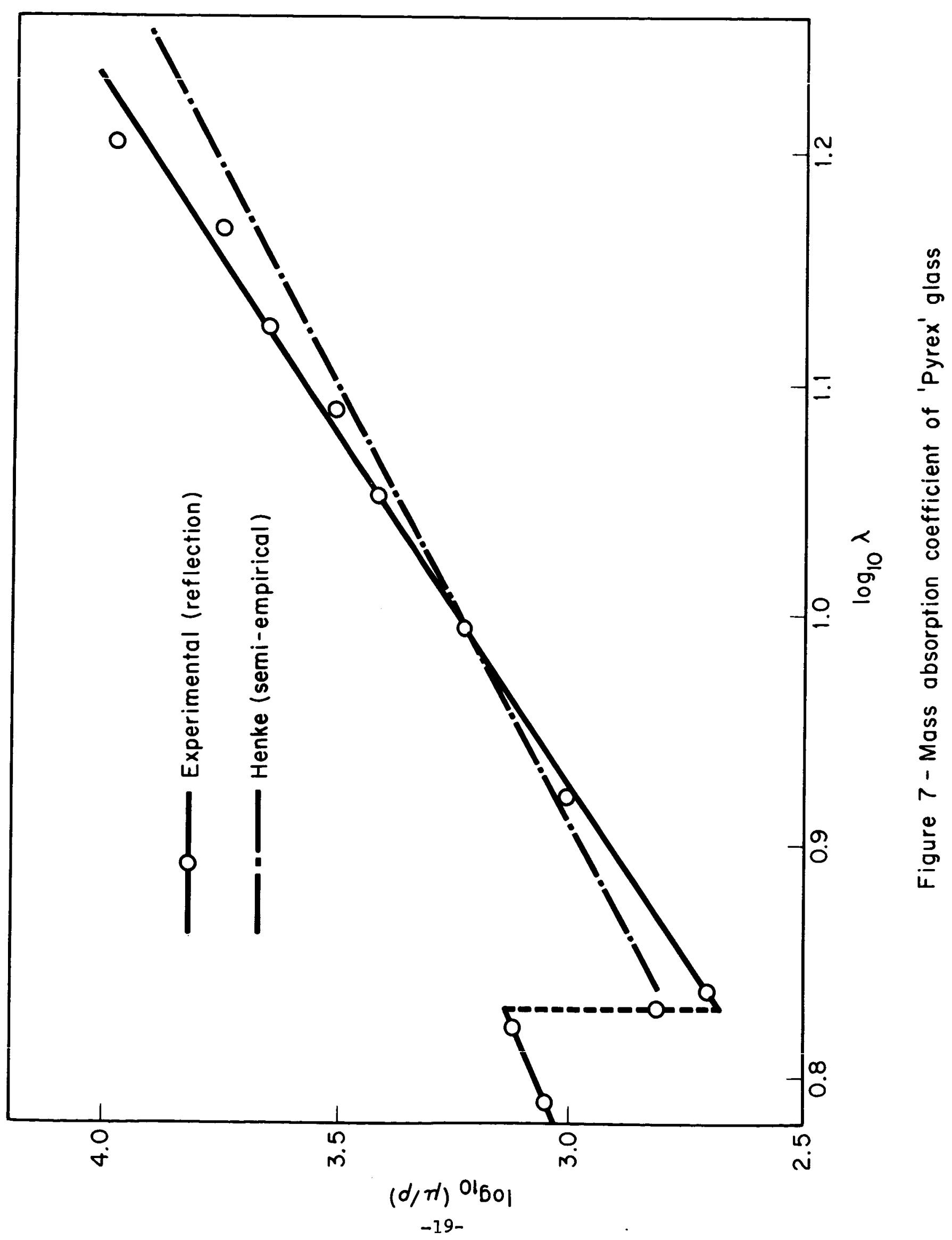




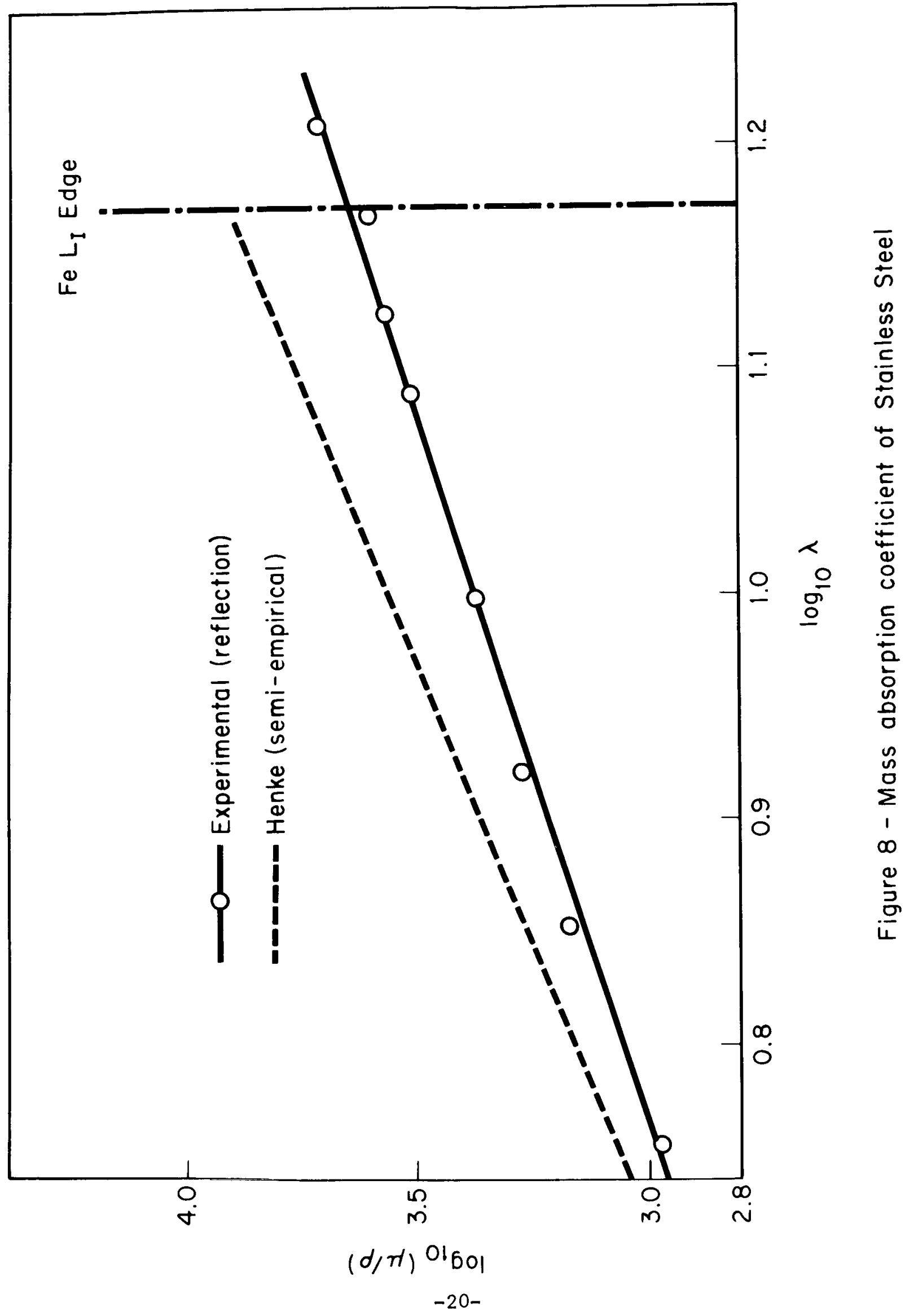


A similar effect, but of a smaller magnitude, occurs for glass (Fig. 9). The theoretical curves are once again calculated using the Kallmann-Mark dispersion theory. The agreement between theory and experiment is improved somewhat if the effective number of electrons in the $K$ shells of silicon and oxygen is assumed to be 1.5 (dashed line) but at the longer wavelengths there is still a discrepancy.

\section{CONCLUSION}

It is not possible, at the present time, to predict accurately the efficiency of $X$-ray reflection of a material at a given angle of incidence, as existing theories of reflection are based on models of perfect surface smoothness, whereas a real surface has a degree of roughness dependent on the method of preparation. At the surface of a real material there is also an electron density gradient which affects the reflection intensity, and at the present time the form and extent of this density gradient can only be determined by experiments on X-ray reflection. Until some other means is developed whereby these two factors may be independently evaluated and their effects predicted, we must reply on experimental and semi-empirical reflection data when designing systems using $\mathrm{X}$-ray mirrors.

\section{ACRNOWLEDGMENT}

The author is indebted to Professor E. A. Stewardson for suggesting this investigation, and for valuable discussions throughout the course of the work. 


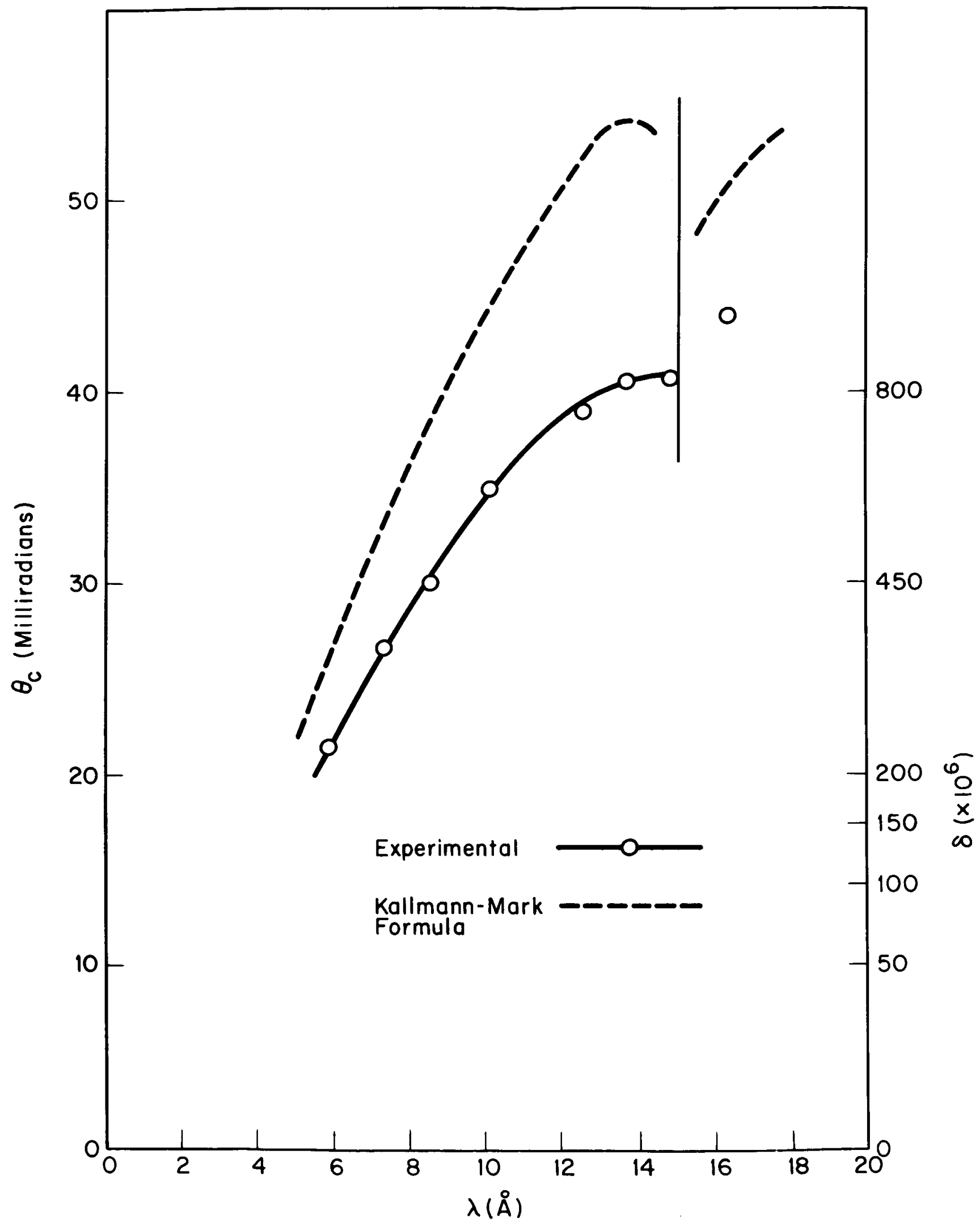

Figure 10 - Dispersion of Stainless Steel 


\section{DEFEDENCES}

1. COMPTON, A. H. and ALLISON, S. K., 1935, X-rays in Theory and Experiment (New York: Van Nostrand)

2. COOKE, B. A., 1963, Thesis, Leicester

3. EHRENBERG, W., 1949, JOSA 39,741

4. GROTH, R., 1959, Ann. Physik, VII 2, 380

5. HENDRICK, R., 1957, JOSA 47,165

6. HENKE, B. L., WHITE, R., and LUNDBERG, B., 1957, J. Appl. Phys. 28, 98

7. HONL, H. 1933, Z. Physik 84, 1

8. KALIMANN, H. and MARK, H., 1927, Ann Physik 82, 385

9. NORDFORS, B., 1961, Thesis, Uppsala

10. PARRATT, L. G., 1954, Phys. Rev. 95, 359

11. PARRATT, L. G. and HEMPSTEAD, C. F., 1954, Phys. Rev. 94, 1593

12. SAURo, J., FANKUCHEN, I. and WAINFAN, N., 1963, Phys. Rev. 132, 1544

13. WUERKER, R. F., 1956, Thesis, Stanford 